\title{
THE GEOMETRY ON THE SLOPE OF A MOUNTAIN
}

\author{
P. CHANSRI, P. CHANSANGIAM, AND SORIN V. SABAU
}

Received 15 January, 2019

\begin{abstract}
The geometry on a slope of a mountain is the geometry of a Finsler metric, called here the slope metric. We study the existence of globally defined slope metrics on surfaces of revolution as well as the geodesic's behavior. A comparison between Finslerian and Riemannian areas of a bounded region is also studied.
\end{abstract}

2010 Mathematics Subject Classification: 53A35; 53C60

Keywords: Finsler metric, slope metric, surface of revolution, geodesic

\section{INTRODUCTION}

Finsler manifolds, that is $n$-dimensional smooth manifolds endowed with Finsler metrics, are natural generalization of the well-known Riemannian manifolds. The main difference is that the metric itself and all Finsler geometric quantities depend not only on the point $x \in M$ of the manifold, but also on the direction $y \in T_{x} M$, where $(x, y)$ are the canonical coordinates of the tangent bundle $T M$. This directional dependence reveals many hidden geometrical features that are usually obscured by the quadratic form in the $y$-variable of a Riemannian metric.

The Randers metrics $F=\alpha+\beta$ and the Kropina metrics $F=\frac{\alpha^{2}}{\beta}$ belong to a larger class of Finsler metrics called $(\alpha, \beta)$ - metrics since they are obtained by deformations of a Riemannian metric by means of a linear 1 -form $\beta=b_{i}(x) y^{i}$ on $T M$. The common characteristic is that they are obtained by rigid translation of a Riemannian unit sphere by a vector field $W$. The local and global geometries of these Finslerian metrics have been extensively studied ([11]).

Another interesting but much less studied problem is the Matsumoto's slope metric $F=\frac{\alpha^{2}}{\alpha-\beta}$. Indeed, based on a letter of P. Finsler (1969), M. Matsumoto considered the following problem:

Suppose a person walking on a horizontal plane with velocity $c$, while the gravitational force is acting perpendicularly on this plane. The person is almost ignorant of the action of this force. Imagine the person walks now with same velocity on the 
inclined plane of angle $\varepsilon$ to the horizontal sea level. Under the influence of gravitational forces, what is the trajectory the person should walk in the center to reach a given destination in the shortest time?

Based on this, he has formulated the following Slope principle ([9] ).

With respect to the time measure, a plane $(\pi)$ with an angle $\varepsilon$ inclination can be regarded as a Minkowski plane. The indicatrix curve of the corresponding Minkowski metric is a limaçon, contained in this plane, given by

$$
r=c+a \cos \theta,
$$

in the polar coordinates $(r, \theta)$ of $(\pi)$, whose pole is the origin $O$ of $(\pi)$ and the polar axis is the most steepest downhill direction, where $a=\frac{g}{2} \sin \varepsilon$, and $g$ is the acceleration constant.

From calculus of variations it follows that for a hiker walking the slope of a mountain under the influence of gravity, the most efficient time minimizing paths are not the Riemannian geodesics, but the geodesics of the slope metric $F=\frac{\alpha^{2}}{\alpha-\beta}$.

More recently, it was shown that the fire fronts evolution can be modeled by Finsler metrics of slope type and their generalizations (see [8]). In this setting the geodesics behaviour and the cut locus have real interpretations and concrete applications for the firefighters activity as well as preventing of wild fires. All these applications show that slope metrics deserve a more detalied study making in this way the motivation of the preseant paper.

Despite the quite long existence of slope metrics, their study is limited mainly to the study of their local geometrical properties, while the global existence of such metrics and other geometrical properties are conspicuously absent.

Our study leads to the following novel findings:

(1) we show that there are many examples of surfaces admitting globally defined slope metrics;

(2) we describe in some detail the geometry of a surface of revolution endowed with a slope metric. In special we study the geodesics behaviour, Clairaut relation, etc.;

(3) we compare the Finslerian areas (by using the Busemann-Hausdorff and the Holmes-Thompson volume forms, respectively) with the Riemannian one.

Here is the contents of the present paper. We recall in Section 2 the construction of the slope metric on a surface $M \rightarrow \mathbb{R}^{3}$ based on Matsumoto's work pointing out the strongly convexity condition such a surface must satisfy in order to admit a slope metric (Proposition 1).

Based on these we show that there exist smooth surfaces $M \rightarrow \mathbb{R}^{3}$ that admit globally defined slope metrics (Section 3). All the examples known until now were local one. This is for the first time the existence of global slope metrics is shown. 
In Section 4 we specialize to surfaces of revolution admitting globally defined slope metrics. We study in Section 4.1 general Finsler surfaces of revolution and give a new form of the Clairaut relation in Theorem 1. This relation is very important showing that the geodesic flow of Finsler surfaces of revolution is integrable despite its highly nonlinear character. After solving the algebraic system (4.6) one can write the geodesic equations in an explicit form, however solving this system is not a trivial task. Next, in Section 4.3, we construct explicitly the slope metric on a surface of revolution and show that there are many such surfaces admitting globally defined strongly convex slope metrics, see Theorem 3 for a topological classification and examples. These are actually Finsler surfaces of revolution (see Theorem 2).

We turn to study of geodesics of slope metrics on a surface of revolution in Section 4.4 by explicitly writing the geodesic equations as second order ODEs in (4.9). Some immediate consequences are given (see Proposition 2, 3). The meridians are $F$ geodesics, but parallels are not. Moreover, a slope metric cannot be projectively flat or projectively equivalent to Riemannian metric $\alpha$ (Proposition 4). We show the concrete form of the Clairaut relation for this case in Theorem 4, and some consequence of it in Proposition 5.

Finally, we compare the area of a bounded region $D$ on the surface of revolution $M$ when measured by the canonical Riemannian, Busemann-Hausdorff, and HolmesThompson volume measures, respectively (see Theorems 5 and 6).

Other topics in the geometry of slope metrics like the study of the flag curvature, global behaviour of geodesics, and cut locus, etc. will be considered in forthcoming research.

\section{THE SLOPE METRIC}

The slope metric is obtained by the movement on a Riemannian surface under the influence of the gravity attraction force. Indeed, assume a hiker is walking on the surface $M$, seen now as the slope of a mountain, with speed $c$ an level ground, along a path that makes an angle $\varepsilon$ with the steepest downhill direction.

Let us consider the surface $M$ embedded in the Euclidean space $\mathbb{R}^{3}$ with the parametrization $M \rightarrow \mathbb{R}^{3}, \quad(x, y) \mapsto(x, y, z=f(x, y))$, where $f: \mathbb{R}^{2} \rightarrow \mathbb{R}$ is a smooth function (further conditions will be added later), that is $M$ is the graph of $z=f(x, y)$. The tangent plane $\pi_{p}=T_{p} M$ at a point $p=(x, y, f(x, y)) \in M$ is spanned by $\partial_{x}:=$ $\left(1,0, f_{x}\right), \quad \partial_{y}:=\left(0,1, f_{y}\right)$, where $f_{x}$ and $f_{y}$ are the partial derivatives of $f$ with respect to $x$ and $y$, respectively. The induced Riemannian metric from $\mathbb{R}^{3}$ to the surface $M$ is

$$
a_{i j}=\left(\begin{array}{cc}
1+f_{x}^{2} & f_{x} f_{y} \\
f_{x} f_{y} & 1+f_{y}^{2}
\end{array}\right) .
$$

Remark 1. Observe that at a critical point $p \in M$, i.e. a point where $\left(f_{x}(p), f_{y}(p)\right)=$ $(0,0)$, the tangent plane $T_{p} M$ is spanned by the unit vectors $(1,0,0)$ and $(0,1,0)$, while the induced Riemannian metric is just the usual flat metric. 
We will construct the slope metric on the surface $M$ by considering the plane $x, y$ to be the sea level, the $z \geq 0$ coordinate to be the altitude above the sea level and the surface $M: z=f(x, y)$ to be the slope of the mountain.

At any point $p \in M$ we construct a Riemannian orthonormal frame $\left\{e_{1}, e_{2}\right\}$ in $T_{p} M$ by choosing $e_{1}$ to point on the steepest downhill direction of $T_{p} M$. Indeed, it is elementary to see that

$$
e_{1}=-\frac{1}{\sqrt{\left(1+f_{x}^{2}+f_{y}^{2}\right)\left(f_{x}^{2}+f_{y}^{2}\right)}}\left(f_{x} \partial_{x}+f_{y} \partial_{y}\right), \quad e_{2}=\frac{1}{\sqrt{f_{x}^{2}+f_{y}^{2}}}\left(-f_{y} \partial_{x}+f_{x} \partial_{y}\right)
$$

is a such orthonormal frame.

With these notations, the Matsumoto's slope principle is telling us that the locus of unit time destinations of the hiker on the plane $T_{p} M$ is given by the limaçon $r=$ $c+a \cdot \cos \theta$, where $(r, \theta)$ are the polar coordinates in $T_{p} M, c$ is the speed of the hiker on the ground level $x y$, and $a=\frac{g}{2} \cdot \sin \varepsilon$ is the gravity (of magnitude $g$ ) component along the steepest downhill direction. The Finsler norm $F$ having this limaçon as indicatrix measures time travel on the surface $S$.

Taking into account the parametrization

$$
X(t)=(c+a \cos t) \cdot \cos t, \quad Y(t)=(c+a \cos t) \cdot \sin t, \quad t \in[0,2 \pi)
$$

it is easy to obtain the implicit equation of the limaçon

$$
X^{2}+Y^{2}=c \sqrt{X^{2}+Y^{2}}+a \cdot X,
$$

where $X, Y$ are the coordinates with respect to the orthonormal frame $\left\{e_{1}, e_{2}\right\}$ in $T_{p} M$.

We get the Minkowski norm $F(X, Y)=\frac{X^{2}+Y^{2}}{c \sqrt{X^{2}+Y^{2}}+a \cdot X}$, and by converting to the canonical coordinates $(x, y, \dot{x}, \dot{y})$ of $T M$ we obtain the slope metric

$$
F(x, y, \dot{x}, \dot{y})=\frac{\alpha^{2}}{c \alpha-\frac{g}{2} \beta},
$$

where

$$
\left\{\begin{array}{l}
\alpha=\sqrt{\left(1+f_{x}^{2}\right) \dot{x}^{2}+2 f_{x} f_{y} \dot{x} \dot{y}+\left(1+f_{y}^{2}\right) \dot{y}^{2}} \\
\beta=f_{x} \dot{x}+f_{y} \dot{y} .
\end{array}\right.
$$

Remark 2. Recall that a Finsler structure $(M, F)$ is a surface (or more generally an $n$-dimensional manifold) $M$ endowed with a Banach norm in each tangent space that smoothly varies with the base point all over the manifold. A Riemannian structure is the particular case when each of these norms are induced by a quadratic form.

Observe that the slope metric as the Finsler metric whose indicatrix is a limaçon, was effectively constructed only at regular points of the surface $M$. However, it is easy to see that the resulting Finsler metric is well defined everywhere on $M$, including the critical points of $M$, where it becomes the flat Riemannian metric. This is in 
perfect accord with the physical situation initially considered, since in these points the gravitation forces have no influence.

For the sake of simplicity we can choose $c:=\frac{g}{2}$ and by multiplication with $c$ we obtain the usual form of the slope metric

$$
F=\frac{\alpha^{2}}{\alpha-\beta}
$$

(see [3], [9]). The slope metric belongs to the class of $(\alpha, \beta)$-metrics (see [1]).

By writing $F=F(\alpha, \beta)=\alpha \cdot \phi(\mathfrak{s})$, where $\mathfrak{s}=\frac{\beta}{\alpha}$, the Hessian $g_{i j}:=\frac{1}{2} \frac{\partial^{2} F^{2}}{\partial y^{i} \partial y^{j}}$ reads $g_{i j}=\rho a_{i j}+\rho_{0} b_{i} b_{j}+\rho_{1}\left(b_{i} \alpha_{j}+b_{j} \alpha_{i}\right)-\rho \rho_{1} \alpha_{i} \alpha_{j}$, where $\alpha_{i}:=\frac{\partial \alpha}{\partial y^{i}}$, and $\rho=\phi^{2}-\mathfrak{s} \phi \phi^{\prime}$, $\rho_{0}=\phi \phi^{\prime \prime}+\phi^{\prime} \phi^{\prime}, \rho_{1}=-\mathfrak{s}\left(\phi \phi^{\prime \prime}+\phi^{\prime} \phi^{\prime}\right)+\phi \phi^{\prime}$.

It is known from Shen's work (see [1]) that $(\alpha, \beta)$ type Finsler metrics are strongly convex whenever the function $\phi(\mathfrak{s})$ satisfies

$$
\phi(\mathfrak{s})>0, \quad \phi(\mathfrak{s})-\mathfrak{s} \phi^{\prime}(\mathfrak{s})>0, \quad \phi^{\prime \prime}(\mathfrak{s}) \geq 0, \quad \text { for } \quad \mathfrak{s}<b .
$$

In the case of the slope metric, we have $\phi(\mathfrak{s})=\frac{1}{1-s}$ and the relations above are clearly satisfied for $\mathfrak{s}<\frac{1}{2}$, that is $\beta<\frac{1}{2} \alpha$. It follows

Proposition 1. A surface $M \rightarrow \mathbb{R}^{3},(x, y) \mapsto(x, y, z=f(x, y))$ admits a strongly convex slope metric $F=\frac{\alpha^{2}}{\alpha-\beta}$, where $\alpha, \beta$ are given in (2.5), if and only if

$$
f_{x}^{2}+f_{y}^{2}<\frac{1}{3}
$$

where $f_{x}, f_{y}$ are partial derivatives of $f$.

This proposition is saying that $\beta<\frac{1}{2} \alpha$ is equivalent to the condition (2.7), for $\alpha, \beta$ given in (2.5).

Remark 3. (1) This formula was obtained for the first time in [3] and the proof is based on the idea in [3].

(2) The convexity formula above is obviously equivalent to the usual convexity condition of the limaçon $c>2 a$.

(3) Taking into account the inverse matrix $\left(a^{i j}\right)$ of (2.1), it can be seen that $b^{2}:=a^{i j} b_{i} b_{j}$ is given by $b^{2}=\frac{f_{x}^{2}+f_{y}^{2}}{1+f_{x}^{2}+f_{y}^{2}}$, and from (2.7) it follows that the strongly convexity of the indicatrix is equivalent to $b<\frac{1}{2}$.

Observe that for the slope metric (2.6) we have $\rho=\frac{2 \mathfrak{s}-1}{(\mathfrak{s}-1)^{3}}=\frac{\alpha^{2}(\alpha-2 \beta)}{(\alpha-\beta)^{3}}, \rho_{0}=$ $\frac{3}{(\mathfrak{s}-1)^{4}}=\frac{3 \alpha^{4}}{(\alpha-\beta)^{4}}, \rho_{1}=\frac{1-4 \mathfrak{s}}{(\mathfrak{s}-1)^{4}}=\frac{\alpha^{3}(\alpha-4 \beta)}{(\alpha-\beta)^{4}}$ and hence $g_{11}=\rho \cdot a_{11}+\rho_{0}+2 \rho_{1} \alpha_{1}-\rho$. $\rho_{1}\left(\alpha_{1}\right)^{2}, g_{12}=\left(1-\rho \alpha_{1}\right) \rho_{1} \alpha_{2}, g_{22}=\rho \cdot a_{22}-\rho \cdot \rho_{1}\left(\alpha_{2}\right)^{2}$. 


\section{EXAMPLES OF SLOPE METRICS}

We will consider in the following some simple examples of slope metrics. Observe that, as pointed already, the base surfaces may have critical points. At these points the slope metric reduces to a flat Riemannian metric, fact in perfect agreement with the initial setting of the problem.

One might be tempted to think that due to the convexity condition (2.7) the slope metric is strongly convex only locally. See for instance the example of the paraboloid of revolution $f(x, y):=100-x^{2}-y^{2}$ in [3] where the strongly convexity condition is assured only in a circular vicinity of the hilltop. However, that is not the case. There are many Riemannian surfaces that admit globally strongly convex slope metric. We describe few such examples below.

\subsection{The plane}

The simplest surface is the plane $M: z=f(x, y)=p x+q y+r$, where $p, q, r$ are constants.

It is trivial to see that

$$
\left(a_{i j}\right)=\left(\begin{array}{cc}
1+p^{2} & p q \\
p q & 1+q^{2}
\end{array}\right), \quad\left(b_{i}\right)=\left(\begin{array}{l}
p \\
q
\end{array}\right)
$$

thus the slope metric is actually the Minkowski metric

$$
F=\frac{\left(1+p^{2}\right) \dot{x}^{2}+2 p q \dot{x} \dot{y}+\left(1+q^{2}\right) \dot{y}^{2}}{\sqrt{\left(1+p^{2}\right) \dot{x}^{2}+2 p q \dot{x} \dot{y}+\left(1+q^{2}\right) \dot{y}^{2}}-(p \dot{x}+q \dot{y})},
$$

with the strongly convexity condition $p^{2}+q^{2}<\frac{1}{3}$. Hence, the plane $z=\lambda \cdot x$ does admit a strongly convex slope metric for any constant $\lambda^{2}<\frac{1}{3}$, while $z=x$ does not (see [3]).

We recall from [12] that for a slope metric on a surface $M: z=f(x, y)$, the 1-form $\beta$ is parallel with respect to $\alpha$ if and only if $M$ is a plane. In this case the slope metric is a Berwald space.

\subsection{A list of surfaces}

Elementary computations show that all the following surfaces $z=f(x, y)$ admit strongly convex slope metric globally defined, where $f: \mathbb{R}^{2} \rightarrow \mathbb{R}$ are given by

(1) $f(x, y)=\frac{1}{2 \sqrt{6}} e^{-\left(x^{2}+y^{2}\right)}$,

(2) $f(x, y)=\frac{1}{2 \sqrt{6}} e^{-(x+y)^{2}}$,

(3) $f(x, y)=\frac{1}{2 \sqrt{6}} \arctan (x+y)$,

(4) $f(x, y)=\frac{1}{2 \sqrt{6}}\left((x+y)-\log \left(e^{x+y}+1\right)\right)$,

(5) $f(x, y)=\frac{1}{2 \sqrt{6}} \log \left(\sqrt{(x+y)^{2}+1}+x+y\right)$. 
Indeed, all these surfaces satisfy condition (2.7) for any $(x, y) \in \mathbb{R}^{2}$.

Let us remark that the surface $f(x, y):=\frac{1}{2 \sqrt{6}} e^{-\left(x^{2}+y^{2}\right)}$ can be actually realized as a surface of revolution obtained by rotating the graph of the function $z=\frac{1}{2 \sqrt{6}} e^{-x^{2}}$ around the $z$ axis. This example suggests that surfaces of revolution are good candidates for the study of slope metrics, fact motivating the next section.

\section{THE SLOPE METRIC OF A SURFACE OF REVOLUTION}

\subsection{Riemannian surface of revolution}

Let us recall some facts from the geometry of Riemannian surfaces of revolution (see [13]).

A surface of revolution $M \rightarrow \mathbb{R}^{3}$ can be parametrization as

$$
(u, v) \mapsto(x=m(u) \cos v, y=m(u) \sin v, z=u)
$$

where $u \in(0, \infty), v \in \mathbb{S}^{1}$. Here $(u, v)$ are the geodesic polar coordinates around the pole $p \in M$, and $m:(0, \infty) \rightarrow(0, \infty)$ is a smooth function such that $m^{\prime}(0)=1$ (see [13] for details).

Remark 4. We have defined here a classical surface of revolution by rotating the image of the curve $m:(0, \infty) \rightarrow(0, \infty)$ around the $z$ axis. However, there is no harm in taking $m: I \rightarrow(0, \infty)$, where $I \subset \mathbb{R}$ is an open set.

It is known that a curve $u=u(t), v=v_{0}$ : constant is called a meridian, and $u=u_{0}$ : constant, $v=v(t)$ is called a parallel. A point $p \in M$ is called pole if any 2 geodesics emanating from $p$ do not meet again, in other words, the cut locus of $p$ is empty. A unit speed geodesic is called a ray if $d(\gamma(0), \gamma(s))=s$, for all $s \geq 0$. Clearly, all geodesics emanating from the pole are rays.

The induced Riemannian metric is

$$
\left(a_{i j}\right)=\left(\begin{array}{cc}
1+\left(m^{\prime}\right)^{2}(u) & 0 \\
0 & m^{2}(u)
\end{array}\right)
$$

and the unit speed geodesics $(u=u(t), v=v(t))$ are given by

$$
\left\{\begin{array}{l}
\frac{d^{2} u}{d t^{2}}+\frac{m^{\prime} m^{\prime \prime}}{1+m^{\prime 2}}\left(\frac{d u}{d t}\right)^{2}-\frac{m m^{\prime}}{1+m^{\prime 2}}\left(\frac{d v}{d t}\right)^{2}=0 \\
\frac{d^{2} v}{d t^{2}}+2 \frac{m^{\prime}}{m} \frac{d u}{d t} \frac{d v}{d t}=0 .
\end{array}\right.
$$

The geodesic spray coefficients of this Riemannian metric read

$$
\left\{\begin{array}{l}
2 \mathcal{G}_{\alpha}^{1}=\frac{m^{\prime} m^{\prime \prime}}{1+m^{\prime 2}}\left(y^{1}\right)^{2}-\frac{m m^{\prime}}{1+m^{\prime 2}}\left(y^{2}\right)^{2} \\
2 \mathcal{G}_{\alpha}^{2}=2 \frac{m^{\prime}}{m} y^{1} y^{2}, \quad(m \neq 0) .
\end{array}\right.
$$

From here it follows that there exists a constant $v$, called the Clairaut constant such that

$$
\frac{d v}{d t} \cdot m^{2}(u(t))=v
$$


and hence $\frac{d u}{d t}= \pm \frac{1}{m} \sqrt{\frac{m^{2}-v^{2}}{1+m^{\prime 2}}}$, that is in the case of a Riemannian surface of revolution, the geodesic flow is integrable.

Remark 5. By changing the parameter $u$ on the profile curve $m(u)$ we can parametrize $M$ as $(u, v) \mapsto(m(u) \cos v, m(u) \sin v, z(u))$ such that $\left[m^{\prime}(u)\right]^{2}+\left[z^{\prime}(u)\right]^{2}=1$. This simplifies the induced Riemannian metric $\left(a_{i j}\right)$. We are not using this parametrization because linear form $\beta$ in (2.5) is simpler when using (4.1) and this leads to simplication of computations for the slope metric.

\subsection{Finsler surfaces of revolution}

Let $(M, F)$ be a Finsler structure defined on a surface of revolution $M$ defined as in Section 4.1.

If $X:=\frac{\partial}{\partial v}$ is a Killing vector field for $F$, that is $\mathcal{L}_{X} F=X^{c}(F)=0$, where $X^{c}$ is the complete lift of $X$ to $T M$, or equivalently $\frac{\partial F}{\partial v}=0$, then $(M, F)$ is called a Finsler surface of revolution.

Remark 6. (1) See [7] for a definition based on the notion of motion. Their definition is equivalent to ours.

(2) See [5] and [6] for a complete study of rotationally Randers metrics, that is Finsler metric of type $F=\alpha+\beta$ constructed on surfaces of revolution.

If we denote by $H(x, p)$ the Hamiltonian corresponding to the Finsler structure $(M, F)$ by means of Legendre transform (see [10]), then since $F$ is surface of revolution, it follows $\frac{\partial H}{\partial v}=0$. Hence, Hamilton Jacobi equations $\frac{d x^{i}}{d s}=\frac{\partial H}{\partial p_{i}}, \frac{d p_{i}}{d s}=-\frac{\partial H}{\partial x^{i}}$ imply that $I=p_{2}$ is a prime integral of the geodesic flow, that is $\frac{d p_{2}}{d s}=0$ along any unit speed $F$-geodesic.

On the other hand, recall that by the Legendre transform associated to $F$, we have $p_{2}=g_{2 i} y^{i}=g_{12} y^{1}+g_{22} y^{2}$ and hence we obtain

Theorem 1. Along any unit speed F-geodesics $\mathcal{P}(s)=(u(s), v(s))$ we have

$$
p_{2}(s)=g_{12}(\mathcal{P}, \dot{\mathcal{P}}) \cdot \frac{d u}{d s}+g_{22}(\mathcal{P}, \dot{\mathcal{P}}) \cdot \frac{d v}{d s}=v_{F}=\text { constant } .
$$

That is, (4.5) is the corresponding relation to (4.4) in the Finslerian setting. The constant $v_{F}$ plays the role of the Clairaut constant for Finslerian geodesics.

Remark 7. See [7] for an alternate proof of this formula.

It follows that, for any unit speed $F$-geodesic, we have

$$
g_{12}(\mathcal{P}, \dot{\mathcal{P}}) \cdot \frac{d u}{d s}+g_{22}(\mathcal{P}, \dot{\mathcal{P}}) \cdot \frac{d v}{d s}=\mathrm{v}, \quad F(\mathcal{P}, \dot{\mathcal{P}})=1
$$

and theoretically, by solving this algebraic system, we can obtain $\frac{d u}{d t}$ and $\frac{d v}{d t}$ that by integration would give the trajectories of the $F$-geodesics. However, observe that finding an explicit solution of the system is not a trivial task. 
Remark 8. (1) As far as we know, the relation (4.5) appeared for the first time in the case of the rotational Randers surface of revolution studied in [5], where the Clairaut constant for the Randers geodesics is $\frac{v}{1+\mu \nu}$. Here $v$ is the usual Clairaut constant of the corresponding Riemannian geodesic through the Zermelo navigation process.

(2) We denote by $\varphi_{t}$ the flow of $\frac{\partial}{\partial v}$, which is a Finslerian isometry preserving the orientation of $M$. The Finslerian distance $d_{F}$ is invariant under $\varphi_{t}$.

\subsection{The slope metric on a surface of revolution}

Let us consider again the surface of revolution $M$ with the parametrization (4.1) and induced Riemannian metric (4.2).

Following again Matsumoto's slope principle, observe that the orthonormal frame in $T_{p} M$ at a given $p \in M$ is $e_{1}=-\frac{1}{\sqrt{\left(m^{\prime}\right)^{2}+1}} \cdot \frac{\partial}{\partial u}, e_{2}=\frac{1}{m} \cdot \frac{\partial}{\partial v}$ and here, the relation between the coordinates $(X, Y)$ of $T_{p} M$ with respect to $\left\{e_{1}, e_{2}\right\}$ and the canonical coordinates $(\dot{u}, \dot{v})$ is $X=-\sqrt{1+\left(m^{\prime}\right)^{2}} \cdot \dot{u}, \quad Y=m \cdot \dot{v}$.

The limaçon implicit equation (2.4) reads now

$$
\left[1+\left(m^{\prime}\right)^{2}\right] \dot{u}^{2}+m^{2} \cdot \dot{v}^{2}=c \sqrt{\left[1+\left(m^{\prime}\right)^{2}\right] \dot{u}^{2}+m^{2} \dot{v}^{2}}-a \sqrt{1+\left(m^{\prime}\right)^{2}} \cdot \dot{u},
$$

and taking into account that $a=\sin \varepsilon=\frac{1}{\sqrt{1+\left(m^{\prime}\right)^{2}}}$ we obtain the slope metric in the form (2.6) with

$$
\alpha=\sqrt{\left[1+\left(m^{\prime}\right)^{2}\right] \dot{u}^{2}+m^{2} \dot{v}^{2}}, \quad \beta=\dot{u} .
$$

Taking into account the strongly convexity condition $b<\frac{1}{2}$ it follows

Theorem 2. A surface of revolution $M \rightarrow \mathbb{R}^{3},(u, v) \mapsto(m(u) \cos v, m(u) \sin v, u)$ admits a strongly convex slope metric $F=\frac{\alpha^{2}}{\alpha-\beta}$, with $\alpha, \beta$ given in (4.7) if and only if

$$
\left(m^{\prime}\right)^{2}>3 \text {. }
$$

Moreover, $(M, F)$ is a Finsler surface of revolution.

Let us recall from Poincaré -Hopf index theorem for the rotational vector filed $X=\left.\frac{\partial}{\partial v}\right|_{p}, p \in M$, that the strongly convexity condition (4.8) implies that number of singular points of $X$ on $M$ can be only 1 or 0 . Indeed, otherwise $X$ would be vanishing, or $M$ would be homeomorphic to the sphere, and this is not possible. It is clear from (4.8) that $M$ cannot be boundaryless compact manifold. The case of a cylinder of revolution is not possible either due (4.8), hence we obtain

Theorem 3. The surfaces of revolution $M$ admitting globally defined strongly convex slope metrics are homeomorphic to $\mathbb{R}^{2}$.

One can now easily construct examples of surfaces of revolution satisfy condition (4.8). Here are such surfaces 
(1) $m(u)=\sqrt{6 u^{2}-1}$, for $u \in\left(\frac{1}{\sqrt{6}}, \infty\right)$;

(2) $m(u)=\frac{1}{2} \sqrt{-2 \ln \left(24 u^{2}\right)}$, for $u \in\left(0, \frac{1}{2 \sqrt{6}}\right)$.

Since the slope metric $F$ is a Finslerian surface of revolution, the theory explained in Section 4.2 applies.

\subsection{The geodesics of a surface of revolution with the slope metric}

In order to study to geodesics of the slope metric $\left(M, F=\frac{\alpha^{2}}{\alpha-\beta}\right)$ we need a formula for the geodesic spray of $F$.

We recall the general formula for an arbitrary $(\alpha, \beta)$-metric

$$
G^{i}=\mathcal{G}_{\alpha}^{i}+\alpha Q s_{0}^{i}+\Theta\left\{-2 Q \alpha s_{0}+r_{00}\right\} \frac{y^{i}}{\alpha}+\Psi\left\{-2 Q \alpha s_{0}+r_{00}\right\} b^{i}
$$

where $G^{i}$ and $\mathcal{G}_{\alpha}^{i}$ denote the spray coefficients for $F$ and $\alpha$, respectively.

Here we use the customary notations:

$$
r_{i j}:=\frac{1}{2}\left(b_{i \mid j}+b_{j \mid i}\right), s_{i j}:=\frac{1}{2}\left(b_{i \mid j}-b_{j: i}\right), s_{j}^{i}:=a^{i k} s_{k j}, s_{j}=b_{i} s_{j}^{i}, b^{i}=a^{i j} b_{j},
$$

and

$$
\begin{aligned}
& Q:=\frac{\phi^{\prime}}{\phi-\mathfrak{s} \phi^{\prime}}, \Psi:=\frac{\phi^{\prime \prime}}{2\left[\phi-\mathfrak{s} \phi^{\prime}+\left(b^{2}-\mathfrak{s}^{2}\right) \phi^{\prime \prime}\right]}, \\
& \Theta:=\frac{\phi-\mathfrak{s} \phi^{\prime}}{2\left[\phi-\mathfrak{s} \phi^{\prime}+\left(b^{2}-\mathfrak{s}^{2}\right) \phi\right]} \cdot \frac{\phi^{\prime}}{\phi}-\mathfrak{s} \Psi=\left[\frac{\phi-\mathfrak{s} \phi^{\prime}}{\phi^{\prime \prime}} \cdot \frac{\phi^{\prime}}{\phi}-\mathfrak{s}\right] \Psi
\end{aligned}
$$

(see [1]).

In the case of $\alpha, \beta$ given in (4.7) we obtain

$$
\left(\begin{array}{l}
b_{1 \mid 1} \\
b_{2 \mid 2}
\end{array}\right)=\left(\begin{array}{c}
\frac{-m^{\prime} m^{\prime \prime}}{1+m^{\prime 2}} \\
\frac{m m^{\prime}}{1+m^{\prime 2}}
\end{array}\right), \quad \begin{aligned}
r_{00} & =-2 \cdot \mathcal{G}_{\alpha}^{1} \\
s_{j}^{i} & =s_{j}=0, \quad b_{1 \mid 2}=0,
\end{aligned}
$$

and hence $G^{i}=\mathcal{G}_{\alpha}^{i}+r_{00}\left[\Theta \frac{y^{i}}{\alpha}+\Psi \cdot b^{i}\right]$.

By taking into account now $\phi(\mathfrak{s})=\frac{1}{1-\mathfrak{s}}$ after some computations we get

$$
\begin{aligned}
& \Psi=\frac{1}{2 b^{2}-3 \mathfrak{s}+1}=\frac{\alpha}{\left(2 b^{2}+1\right) \alpha-3 \beta} \\
& \Theta=\left(\frac{1}{2}-2 \mathfrak{s}\right) \Psi=\frac{1-4 \mathfrak{s}}{2\left(2 b^{2}-3 \mathfrak{s}+1\right)}=\frac{\alpha-4 \beta}{2 \alpha} \cdot \Psi=\frac{\alpha-4 \beta}{2\left[\left(2 b^{2}+1\right) \alpha-3 \beta\right]}
\end{aligned}
$$

therefore $G^{i}=\mathcal{G}_{\alpha}^{i}+r_{00}\left[\frac{\alpha-4 \beta}{2 \alpha} \cdot \frac{y^{i}}{\alpha}+b^{i}\right] \cdot \Psi$. 
In particular $G^{1}=\mathcal{G}_{\alpha}^{1} \cdot \frac{(\alpha-2 \beta)^{2}}{\alpha\left[\left(2 b^{2}+1\right) \alpha-3 \beta\right]}, G^{2}=\mathcal{G}_{\alpha}^{2}-\mathcal{G}_{\alpha}^{1} \cdot \frac{\alpha-4 \beta}{\alpha\left[\left(2 b^{2}+1\right) \alpha-3 \beta\right]} \cdot y^{2}$, and therefore, the unit speed $F$-geodesic equations are

$$
\left\{\begin{array}{l}
\frac{d^{2} u}{d s^{2}}+\left.2 \mathcal{G}_{\alpha}^{1} \cdot \frac{(\alpha-2 \beta)^{2}}{\alpha\left[\left(2 b^{2}+1\right) \alpha-3 \beta\right]}\right|_{(u(s), v(s))}=0 \\
\frac{d^{2} v}{d s^{2}}+2 \mathcal{G}_{\alpha}^{2}-\left.2 \mathcal{G}_{\alpha}^{1} \cdot \frac{\alpha-4 \beta}{\alpha\left[\left(2 b^{2}+1\right) \alpha-3 \beta\right]} \cdot \frac{d v}{d s}\right|_{(u(s), v(s))}=0 .
\end{array}\right.
$$

The geodesic equations in this form are not of much use.

However, some conclusions can be drawn.

Proposition 2. The meridians are F-unit speed geodesics.

Proof. If we consider an ( $F$-unit speed) meridian $\mathcal{P}(s)=\left(u(s), v_{0}\right)$, then $\dot{\mathcal{P}}(s)=$ $\left(\frac{d u}{d s}, 0\right)$ and by using the $F$-unit speed condition the geodesic equation (4.9) are identically satisfied.

Proposition 3. A parallel $\mathcal{P}(s)=\left(u_{0}, v(s)\right)$ is $F$-geodesic if and only if $m^{\prime}\left(u_{0}\right)=0$, that is a strongly convex slope metric do not admit parallels geodesics.

Proof. $(\Rightarrow)$ If the parallel $\mathcal{P}(s)=\left(u_{0}, v(s)\right)$ is a unit speed $F$-geodesic, then along $\mathcal{P}(s),\left.\alpha^{2}\right|_{(\mathcal{P}, \dot{P})}=1$ and $\left.\beta\right|_{(\mathcal{P}, \dot{P})}=0$, hence the conclusion follows from the same arguments as in the Riemannian case.

$(\Leftarrow)$ If we assume $m^{\prime}\left(u_{0}\right)=0$ then the conclusion follows in a similar way with the Riemannian case.

Proposition 4. The slope metric $F=\frac{\alpha^{2}}{\alpha-\beta}$ can not be projectively equivalent to the Riemannian metric of $M$, nor projectively flat.

Proof. Recall that a Matsumoto metric $F=\frac{\alpha^{2}}{\alpha-\beta}$ is projectively equivalent to the Riemannian metric $\alpha$ if and only if $\beta$ is parallel with respect to $a_{i j}$, that is $b_{i \mid j}=0$, where $\mid$ is the covariant derivative with respect to $a_{i j}$.

However, observe that in the case of the slope metric we have $b_{1 \mid 1}=-\gamma_{11}^{1} \neq 0$, $b_{1 \mid 2}=0, \quad b_{2 \mid 2}=-\gamma_{22}^{1} \neq 0$.

In order to be projectively flat $\beta$ must be parallel and $\alpha$ projectively flat. Clearly, none of these conditions is true in the case of the slope metric.

Let us consider the prime integral $p_{2}$ of the geodesic flow. A straightforward computation shows

Theorem 4. Along the unit speed $F$-geodesic $\mathcal{P}:(0, a) \rightarrow M, \mathcal{P}(s)=(u(s), v(s))$, $\frac{d u}{d s} \neq 0$ for $s \in(0, a)$ we have:

$$
p_{2}(s)=\left.\left(g_{12} y^{1}+g_{22} y^{2}\right)\right|_{(\mathcal{P}, \dot{P})}=\left.\rho\right|_{(\mathcal{P}, \dot{P})} \cdot m^{2}(u(s)) \cdot \frac{d v}{d s}=v_{F} .
$$


Therefore $\frac{d u}{d s}, \frac{d v}{d s}$ are solutions of the following algebraic system:

$$
\left.\rho\right|_{(\mathcal{P}, \dot{P})} \cdot m^{2}(u(s)) \cdot \frac{d v}{d s}=v_{F},\left.\frac{\alpha^{2}}{\alpha-\beta}\right|_{(\mathcal{P}, \dot{P})}=1,
$$

where $\rho(\mathcal{P}(s), \dot{\mathcal{P}}(s))=\left.\frac{\alpha-2 \beta}{(\alpha-\beta)^{2}}\right|_{(\mathcal{P}(s), \dot{\mathcal{P}}(s))}$. An explicit solution of this algebraic system can be obtained by solving a $4^{\text {th }}$ order equation but the computation is too complicated to be given here.

Instead of writing the explicit solution of (4.11) we point out some consequence of (4.10).

If a unit speed $F$-geodesic $\mathcal{P}(0, a) \rightarrow M$ is tangent to the Killing vector field at its end points, that is

$$
\dot{\mathcal{P}}(0)=\left.\frac{1}{F\left(\mathcal{P}(0),\left.\frac{\partial}{\partial v}\right|_{\mathcal{P}(0)}\right)} \cdot \frac{\partial}{\partial v}\right|_{\mathcal{P}(0)}, \dot{\mathcal{P}}(a)=\left.\frac{1}{F\left(\mathcal{P}(a),\left.\frac{\partial}{\partial v}\right|_{\mathcal{P}(a)}\right)} \cdot \frac{\partial}{\partial v}\right|_{\mathcal{P}(a)},
$$

and $\dot{P}(s)$ is linearly dependent with $\left.\frac{\partial}{\partial v}\right|_{\mathcal{P}(s)}$ for any $s \in(0, a)$, then Clairaut relation (4.10) implies

Proposition 5. If $\mathcal{P}:(0, a) \rightarrow M, \mathcal{P}(s)=(u(s), v(s))$ is an $F$-unit speed geodesic such that $\dot{\mathcal{P}}(0)$ and $\dot{\mathcal{P}}(a)$ are linear dependent vectors with $\left.\frac{\partial}{\partial v}\right|_{\mathcal{P}(0)}$ and $\left.\frac{\partial}{\partial v}\right|_{\mathcal{P}(a)}$, respectively, then $m(u(0))=m(u(a))=v_{F}$. Moreover, $m(u(s))>m(u(0))$ for $s \in(0, a)$.

\section{FINSLERIAN VOLUMES}

It is known that the Euclidean volume form in $\mathbb{R}^{n}$ is the $n$-form $d V_{\mathbb{R}^{n}}:=d x^{1} d x^{2} \ldots d x^{n}$, and the Euclidean volume of a bounded open set $D \subset \mathbb{R}^{n}$ is given by $\operatorname{Vol}(D)=$ $\int_{D} d V_{\mathbb{R}^{n}}=\int_{D} d x^{1} d x^{2} \ldots d x^{n}$.

Obviously, if $D \subset \mathbb{R}^{n}$ is a bounded open set, $\operatorname{Vol}(D)$ is a finite constant.

More generally, let us consider a Riemannian manifold $(M, g)$ with the Riemannian volume form $d V_{g}:=\sqrt{g} d x^{1} d x^{2} \ldots d x^{n}$, and hence the Riemannian volume of $(M, g)$ can be computed as

$$
\operatorname{Vol}(M, g)=\int_{M} d V_{g}=\int_{M} \sqrt{g} d x^{1} d x^{2} \ldots d x^{n}=\int_{M} \theta^{1} \theta^{2} \ldots \theta^{n},
$$

where $\left\{\theta^{1}, \theta^{2}, \ldots, \theta^{n}\right\}$ is a $g$-orthonormal co-frame on $M$, and $g=\operatorname{det}\left(g_{i j}\right)$.

In general, a volume form $d \mu$ on an $n$-dimensional Finsler manifold $(M, F)$ is a globally defined, non-degenerate $n$-form on $M$. In local coordinates we can always write $d \mu=\sigma(x) d x^{1} \wedge \cdots \wedge d x^{n}$, where $\sigma$ is a positive function on $M$.

The usual Finslerian volumes are obtain by different choices of the function $\sigma(x)$. Here are two of the most well studied Finslerian volumes. 
The Busemann-Hausdorff volume form is defined as $d V_{B H}:=\sigma_{B H}(x) d x^{1} \wedge \cdots \wedge$ $d x^{n}$, where

$$
\sigma_{B H}(x):=\frac{\operatorname{Vol}\left(\mathbb{B}^{n}(1)\right)}{\operatorname{Vol}\left(\mathcal{B}_{x}^{n} M\right)},
$$

here $\mathbb{B}^{n}(1)$ is the Euclidean unit $n$-ball, $\mathcal{B}_{x}^{n} M=\{y: F(x, y)=1\}$ is the Finslerian ball and $\mathrm{Vol}$ the canonical Euclidean volume.

This volume form allows us to define the Busemann-Hausdorff volume of the Finsler manifold $(M, F)$ by $\operatorname{vol}_{B H}(M, F)=\int_{M} d V_{B H}$.

Remark 9. Observe that the $n$-ball Euclidean volume is

$$
\operatorname{Vol}\left(\mathbb{B}^{n}(1)\right)=\frac{1}{n} \operatorname{Vol}\left(\mathbb{S}^{n-1}\right)=\frac{1}{n} \operatorname{Vol}\left(\mathbb{S}^{n-2}\right) \int_{0}^{\pi} \sin ^{n-2}(t) d t .
$$

Another volume form naturally associated to a Finsler structure is the HolmesThompson volume form defined by $d V_{H T}=\sigma_{H T}(x) d x^{1} \ldots d x^{n}$, where

$$
\sigma_{H T}(x):=\frac{\operatorname{Vol}\left(\mathcal{B}_{x}^{n} M, g_{x}\right)}{\operatorname{Vol}\left(\mathbb{B}^{n}(1)\right)}=\frac{1}{\operatorname{Vol}\left(\mathbb{B}^{n}(1)\right)} \int_{\mathcal{B}_{x}^{n} M}\left(\operatorname{det} g_{i j}(x, y)\right) d y^{1} \ldots d y^{n},
$$

and the Holmes-Thompson volume of the Finsler manifold $(M, F)$ is defined as $\operatorname{vol}_{H T}(M, F)=$ $\int_{M} d V_{H T}$

Remark 10. If $(M, F)$ is an absolute homogeneous Finsler manifold, then the Busemann-Hausdorff volume is a Hausdorff measure of $M$, and therefore we have $\operatorname{vol}_{B H}(M, F) \geq \operatorname{vol}_{H T}(M, F)$ (see [4]). If $(M, F)$ is not absolute homogeneous, then the inequality above is not true anymore.

In the case of an Finsler $(\alpha, \beta)$-metric, one can compute explicitly the Finslerian volume in terms of the Riemannian volume (see [1]). Indeed, if $(M, F(\alpha, \beta))$ is an $(\alpha, \beta)$-metric on an $n$-dimensional manifold $M$, one denotes

$$
f(b):=\frac{\int_{0}^{\pi} \sin ^{n-2}(t) d t}{\int_{0}^{\pi} \frac{\sin ^{n-2}(t)}{\phi(b \cos (t))^{n}} d t}, \quad g(b):=\frac{\int_{0}^{\pi} \sin ^{n-2}(t) T(b \cos t) d t}{\int_{0}^{\pi} \sin ^{n-2}(t) d t},
$$

where $F=\alpha \phi(\mathfrak{s}), \mathfrak{s}=\beta / \alpha$, and $T(\mathfrak{s}):=\phi\left(\phi-\mathfrak{s} \phi^{\prime}\right)^{n-2}\left[\left(\phi-\mathfrak{s} \phi^{\prime}\right)+\left(b^{2}-\mathfrak{s}^{2}\right) \phi^{\prime \prime}\right]$.

Then the Busemann-Hausdorff and Holmes-Thompson volume forms are given by $d V_{B H}=f(b) d V_{\alpha}$, and $d V_{H T}=g(b) d V_{\alpha}$, respectively, where $d V_{\alpha}$ is the Riemannian volume form.

It is remarkable that if the function $T(\mathfrak{s})-1$ is an odd function of $\mathfrak{s}$, then $d V_{H T}=$ $d V_{\alpha}$. This is the case of Randers metrics (see [2]), but not the case of the slope metric.

The following lemma is elementary.

Lemma 1. Let us consider the following functions

(1) $f:\left(0, \frac{1}{2}\right) \rightarrow\left(\frac{8}{9}, 1\right), f(b):=\frac{2}{2+b^{2}}$,

(2) $g:\left(0, \frac{1}{2}\right) \rightarrow\left(\frac{5 \sqrt{3}}{9}, 1\right), g(b):=\frac{\left(2-3 b^{2}\right)}{2\left(1-b^{2}\right) \sqrt{1-b^{2}}}$, 
(3) $h:\left(0, \frac{1}{2}\right) \rightarrow\left(1, \frac{5 \sqrt{3}}{8}\right), h(b):=\frac{\left(2+b^{2}\right)\left(2-3 b^{2}\right)}{4\left(1-b^{2}\right) \sqrt{1-b^{2}}}$.

Then, $f$ and $g$ are both monotone decreasing while $h$ is monotone increasing on the given intervals.

A direct application of this lemma is the following theorem.

Theorem 5. Let $(M, F)$ be a slope metric on a surface of revolution. Then

$$
\operatorname{Area}_{B H}(D)<\operatorname{Area}_{H T}(D)<\operatorname{Area}_{\alpha}(D)
$$

for any bounded region $D \subset M$.

Proof. Firstly, observe that in the case of a slope metric, formulas (5.3) imply

$$
f(b)=\frac{\pi}{\int_{0}^{\pi}(1-b \cos t)^{2} \cdot d t}=\frac{2}{2+b^{2}}, \quad g(b)=\frac{\left(2-3 b^{2}\right)}{2\left(1-b^{2}\right) \sqrt{1-b^{2}}} .
$$

It results

$$
\begin{aligned}
& d V_{B H}=f(b) d V_{\alpha}=\frac{2}{2+b^{2}} \cdot d V_{\alpha}, \\
& d V_{H T}=g(b) d V_{\alpha}=\frac{\left(2-3 b^{2}\right)}{2\left(1-b^{2}\right) \sqrt{1-b^{2}}} \cdot d V_{\alpha}, \\
& d V_{H T}=h(b) d V_{B H}=\frac{\left(2+b^{2}\right)\left(2-3 b^{2}\right)}{4\left(1-b^{2}\right) \sqrt{1-b^{2}}} \cdot d V_{B H},
\end{aligned}
$$

so the meaning of the function $h$ in Lemma 1 is clear now.

By taking into account the monotonicity of $f, g, h$ described in Lemma 1 , the inequalities stated above hold good.

Moreover, from Lemma 1 we have

Theorem 6. Let $(M, F)$ be a slope metric on a surface of revolution. Then

(1) $\frac{8}{9} \operatorname{Area}_{\alpha}(D) \leq \operatorname{Area}_{B H}(D) \leq \operatorname{Area}_{\alpha}(D)$,

(2) $\frac{5 \sqrt{3}}{9} \operatorname{Area}_{\alpha}(D) \leq \operatorname{Area}_{H T}(D) \leq \operatorname{Area}_{\alpha}(D)$,

(3) $\operatorname{Area}_{B H}(D) \leq \operatorname{Area}_{H T}(D) \leq \frac{5 \sqrt{3}}{8} \operatorname{Area}_{B H}(D)$,

for any bounded region $D \subset M$.

\section{ACKNOWLEDGEMENT}

The authors are grateful to the referee's criticism that have improved the quality of the paper. We express our thanks to Prof. H. Shimada for several discussions on the subject.

This research was supported by King Mongkut's Institute of Technology Ladkrabang Research Fund. grant no. KREF046201. 


\section{REFERENCES}

[1] S. Bácsó, X. Cheng, and Z. Shen, "Curvature properties of $(\alpha, \beta)$-metrics," in Finsler geometry, Sapporo 2005-in memory of Makoto Matsumoto, ser. Adv. Stud. Pure Math. Math. Soc. Japan, Tokyo, 2007, vol. 48, pp. 73-110.

[2] D. Bao, S.-S. Chern, and Z. Shen, An introduction to Riemann-Finsler geometry, ser. Graduate Texts in Mathematics. Springer-Verlag, New York, 2000, vol. 200. [Online]. Available: https://doi.org/10.1007/978-1-4612-1268-3. doi: 10.1007/978-1-4612-1268-3

[3] D. Bao and C. Robles, "Ricci and flag curvatures in Finsler geometry," in A sampler of RiemannFinsler geometry, ser. Math. Sci. Res. Inst. Publ. Cambridge Univ. Press, Cambridge, 2004, vol. 50, pp. 197-259.

[4] C. E. Durán, "A volume comparison theorem for Finsler manifolds," Proc. Amer. Math. Soc., vol. 126, no. 10, pp. 3079-3082, 1998, doi: 10.1090/S0002-9939-98-04629-2. [Online]. Available: https://doi.org/10.1090/S0002-9939-98-04629-2

[5] R. Hama, P. Chitsakul, and S. V. Sabau, "The geometry of a Randers rotational surface," Publ. Math. Debrecen, vol. 87, no. 3-4, pp. 473-502, 2015, doi: 10.5486/PMD.2015.7395. [Online]. Available: https://doi.org/10.5486/PMD.2015.7395

[6] R. Hama, P. Chitsakul, and S. V. Sabau, "The cut locus of a randers rotational 2-sphere of revolution," Publ. Math. Debrecen, vol. 93, no. 3-4, pp. 387-412, 2018.

[7] N. Innami, T. Nagano, and K. Shiohama, "Geodesics in a Finsler surface with one-parameter group of motions," Publ. Math. Debrecen, vol. 89, no. 1-2, pp. 137-160, 2016, doi: 10.5486/PMD.2016.7442. [Online]. Available: https://doi.org/10.5486/PMD.2016.7442

[8] S. Markvorsen, "A Finsler geodesic spray paradigm for wildfire spread modelling," Nonlinear Anal. Real World Appl., vol. 28, pp. 208-228, 2016, doi: 10.1016/j.nonrwa.2015.09.011. [Online]. Available: https://doi.org/10.1016/j.nonrwa.2015.09.011

[9] M. Matsumoto, "A slope of a mountain is a Finsler surface with respect to a time measure," $J$. Math. Kyoto Univ., vol. 29, no. 1, pp. 17-25, 1989, doi: 10.1215/kjm/1250520303. [Online]. Available: https://doi.org/10.1215/kjm/1250520303

[10] R. Miron, D. Hrimiuc, H. Shimada, and S. V. Sabau, The geometry of Hamilton and Lagrange spaces, ser. Fundamental Theories of Physics. Kluwer Academic Publishers Group, Dordrecht, 2001, vol. 118

[11] S. V. Sabau, K. Shibuya, and R. Yoshikawa, "Geodesics on strong Kropina manifolds," Eur. J. Math., vol. 3, no. 4, pp. 1172-1224, 2017, doi: 10.1007/s40879-017-0189-6. [Online]. Available: https://doi.org/10.1007/s40879-017-0189-6

[12] H. Shimada and S. Sabau, "Introduction to matsumoto metric," Nonlinear Anal., vol. 63, pp. 165168, 2005, doi: 10.1016/j.na.2005.02.062.

[13] K. Shiohama, T. Shioya, and M. Tanaka, The geometry of total curvature on complete open surfaces, ser. Cambridge Tracts in Mathematics. Cambridge University Press, Cambridge, 2003, vol. 159. [Online]. Available: https://doi.org/10.1017/CBO9780511543159. doi: 10.1017/CBO9780511543159

\section{Authors' addresses}

\section{P. Chansri}

King Mongkut's Institute of Technology Ladkrabang, Faculty of Science, Department of Mathematics, Chalongkrung Road, Ladkrabang, Bangkok, 10520, Thailand

E-mail address: chansri38416@gmail.com 
P. Chansangiam

King Mongkut's Institute of Technology Ladkrabang, Faculty of Science, Department of Mathematics, Chalongkrung Road, Ladkrabang, Bangkok, 10520, Thailand

E-mail address: pattrawut.ch@kmitl.ac.th

\section{Sorin V. Sabau}

Tokai University, Department of Biological Sciences, 005-8600 Sapporo, Japan

E-mail address: sorin@tokai.ac.jp 